\title{
Comparison of Hybrid Firefly Algorithms for Power Allocation in a TV White Space Network
}

\author{
Kennedy K. Ronoh \\ School of Computing and \\ Informatics \\ University of Nairobi \\ Nairobi, Kenya
}

\author{
George Kamucha \\ Department of Electrical and \\ Information Engineering \\ University of Nairobi \\ Nairobi, Kenya
}

\author{
Tonny K. Omwansa \\ School of Computing and \\ Informatics \\ University of Nairobi \\ Nairobi, Kenya
}

\begin{abstract}
TV white spaces (TVWS) can be used by Secondary Users (SUs) through Dynamic Spectrum Access (DSA) as long as they do not cause harmful interference to Primary Users (PUs). Due spectrum scarcity, there is increasing demand for DSA. When there is a high density of SUs in a TVWS network such as cellular access to TVWS, problem of interference among SUs will arise. Possibility of harmful interference to PUs may also arise. Optimization of power allocation is therefore necessary to reduce the level of interference among SUs and to protect PUs against harmful interference. Performance of different hybrid firefly algorithm with particle swarm optimization and genetic algorithm for optimization of power allocation in a TVWS network are compared. Simulation was done using Matlab. Simulation results show that hybrid of firefly algorithm, particle swarm optimization and genetic algorithm outperform other hybrid firefly algorithms. Hybrid of firefly algorithm, particle swarm optimization and genetic algorithm achieves best throughput, sum power as well as objective function value.
\end{abstract}

\section{General Terms}

Optimization, population based metaheuristic algorithms, evolutionary algorithms, swarm intelligence algorithms.

\section{Keywords}

TV White Spaces, power allocation, cognitive radio, hybrid firefly algorithm, continuous optimization, firefly algorithm, genetic algorithm, particle swarm optimization.

\section{INTRODUCTION}

Spectrum occupancy assessments done in USA, Spain, Singapore, New Zealand and Germany [1] and UK[2] indicate that a large portion of spectrum assigned to PUs is underutilized. Spectrum is considered a scarce resource. More and more devices want a pie of the spectrum and yet the useful spectrum is limited. Dynamic Spectrum Access (DSA) through the use of cognitive radio (CR) techniques is currently being embraced as a solution to spectrum underutilization and spectrum scarcity. This is because DSA, together with $\mathrm{CR}$, provides an efficient way for spectrum management and spectrum sharing. With DSA, spectrum allocated for exclusive use to a PU but not being used by the PU (incumbent), or any other idle frequency bands (such as guard bands) can be shared by different SUs as long as the interference to the incumbent by the secondary users to the PU is kept to an acceptable level [3]. The spectrum band which has attracted a lot interest in the DSA community is the TV White Spaces (TVWS). TVWS is the spectrum band not being utilized efficiently by TV transmitters in the Ultra High Frequency (UHF) band [4]. The main reason for this increased interest is the good propagation characteristics of the sub-

\section{$1 \mathrm{GHz}$ spectrum.}

Regulatory authorities worldwide have mandated the use of geo-location database (GLDB) for protection of incumbent users in TVWS network. GLDB is used by a SU or White Space Device (WSD) to find the set of frequency channels that can be used on a secondary basis at a given area and at any given time [5], [6], [7]. GLDB is populated through the use of a propagation model. The database contains estimated power levels of PUs for any point in a particular region of interest. The WSD, which has a Cognitive Radio System (CRS), queries a central database. The WSD provides the database with parameters such as its location, device type and antenna height. The GLDB will then use this information along with the parameters of all surrounding TV transmitters such as antenna height, transmit power and frequency of operation in order to come up with the list of available TVWS channels that can be used by the WSD on secondary basis without causing harmful interference to the PUs. The GLDB will also give the WSD limits on the transmit power and also the time period in which each channel can be used.

In order to improve Quality of Service (QoS) in a TVWS network and to ensure protection of PU, there is need to optimize power allocation. The objective of this paper is to compare the performance of hybrid firefly algorithms (FA) for power allocation in a TVWS network. Among other population-based metaheuristic algorithms, FA is chosen for power allocation because it has been found to perform better than other algorithms in terms of solution quality and convergence time [8] [9]. Despite its superior performance over other algorithms, FA can get trapped in local optimum. Hybridizing FA with other algorithms enables FA to avoid being trapped in a local optimum. The algorithms to be compared are hybrid FA with genetic algorithm (GA), hybrid FA with particle swarm optimization (PSO) as well as hybrid FA, GA and PSO. Simulation results show that hybrid FA, PSO and GA results in better spectrum allocation as measured by SU sum throughput.

The rest of the paper is organized as follows. Section 2 provides a review of related work on power allocation in a TVWS network and hybrid firefly algorithms. System model and simulation set up are presented in section 3 and 4, respectively. Performance evaluation of the algorithms is discussed in section 5. The paper is concluded in section 6 .

\section{RELATED WORK}

This section presents a review of related work on spectrum allocation as well related work on relevant hybrid firefly algorithms. 


\subsection{Related work on Power Allocation in TVWS}

In [7], we proposed a firefly algorithm based power allocation algorithm in a TVWS network which makes use of a GLDB and that considers interference constraints at both PU and SUs. To ensure adequate protection of PUs, both cochannel and adjacent channel interference are considered. In this paper, we compare the performance of the proposed algorithm with other hybrid FA algorithms.

Our previous work in [10] presents the used of modified firefly algorithm for joint power and spectrum allocation for a TVWS network. FA is modified to solve a continuousdiscrete optimization problem. In this work, we compare the performance of hybrid algorithms for power allocation only.

In [11], transmit power control for a TV white space wireless network consisting of a base station has been investigated. Lagrange multiplier is used to optimize power allocation. Lagrange multiplier method used is not computationally efficient. This is because Lagrange multiplier method is an exact optimization and is not suitable for NP hard problems.

In [12], GLDB based, heuristic iterative power control algorithm with co-channel and adjacent channel interference considerations for multiple device-to-device (ad hoc) TV white space network has been proposed. The objective of the proposed algorithm is to maximize total system throughput through power control on each device to device link while considering interference from SUs to PUs, from PUs to SUs and between SUs.

A detailed method of calculating the maximum permitted emission levels for WSDs has been presented in [13]. The proposed method provides a way to calculate location specific maximum power based on location probability. The proposed method makes use of DTT network planning models in order to provide the GLDB with the needed parameters to perform the necessary calculations. The use location probability is not suitable for optimization of resource allocation for all existing SUs in a network because it does not allow for approximation aggregate interference. It considers only a single SU. It is also applicable when fading is considered. Fading is not considered in this paper.

Power control for a device-to-device network has been studied in [14]. In a device-to-device network, devices communicate directly between themselves without going through the base station. A heuristic iterative power control algorithm with cochannel and adjacent channel interference considerations has been proposed. Interference constraints at both PUs and SUs are considered. The objective of the proposed algorithm is to maximize total system throughput through power control on each device to device link while considering interference from SUs to PUs, from PUs to SUs and between SUs. Lagrange multiplier method used is not computationally efficient. This is because it is an exact algorithm.

\subsection{Hybrid Firefly Algorithm and Particle Swarm Optimization}

A hybrid FA and PSO for problem of combined economic and emission dispatch including valve point effect has been proposed by Arunacham et. al. in [15]. There is no alteration to firefly algorithm in the proposed algorithm but the initial solution that is used by FA is obtained from PSO. According to the authors, the quality of the final solution of FA depends on the initial solution that FA starts with. Simulation results show that hybrid the algorithm performs better than both PSO and FA.

A hybrid FA and PSO algorithm for detection of bundle branch block has been proposed in [16]. The concepts of personal best and global best are added into FA. All the steps in FA remain the same except that firefly movement is changed to incorporate the idea of personal best and global best. Firefly movement involves a move towards the local best $\left(\mathrm{P}_{\mathrm{i}}\right)$ and global best $\left(\mathrm{P}_{\mathrm{g}}\right)$ in the proposed algorithm.

\subsection{Hybrid Firefly Algorithm and Genetic Algorithm}

Rahmani A. and Mirhassani S.A. [17] proposed a hybrid FA and GA. All the steps in the FA remain the same except that in every iteration, the two current best solutions are crossed over. Out of the four offsprings, two best offsprings are then selected. For mutation, one of the two offsprings is randomly selected. If the selected offspring has a better solution compared to the current best solution of FA, it replaces the current best solution. The use of crossover in FA improves FA's exploration ability.

Luthra J. and Pal Saibal K. [18] also proposed a hybrid FA and GA for the solution of the monoalphabetic substitution cipher. Movement of fireflies in space is done using genetic operators and the concept of dominant gene cross over.

\subsection{Hybrid Firefly Algorithm Particle Swarm Optimization and Genetic Algorithm}

Our previous work in [19] presents the use of hybrid FA, PSO and GA for joint power and spectrum allocation for a TVWS network. In this work, we compare the performance of hybrid FA algorithms for power allocation only as a continuous optimization problem. In [19], we did not compare the performance of hybrid FA, PSO and GA with other hybrid FA algorithms.

\subsection{Other Hybrid Firefly Algorithms}

There also exists other hybrid FA with other population based metaheuristic algorithms. A hybrid of bat algorithm and FA has been proposed by Warangal et al.in [20]. A hybrid of cuckoo search of FA has been proposed by Elkhechafi et al. in [21]. A hybrid of FA and ant colony optimization has been proposed by Layeb and Benayad in [22]. PSO and GA are chosen to be hybridized with FA because they are able to converge to a good solution more quickly compared to other population based metaheuristic algorithms

\section{SYTEM MODEL}

The optimization problem to be considered is about power allocation optimization described in our paper in [7]. A network illustrated by Figure 1 is considered. In the figure there a single $\mathrm{TV}$ receiver placed at the edge of the protection region. Among all the TV receivers in the protection region, a $\mathrm{TV}$ receiver at this location is the one which is most vulnerable to interference since it is very close to the secondary network. GLDB regulations require that the protection ratio be measured at the edge of protection region [23]. Aggregate interference at the TV receiver, both cochannel and adjacent channel should not make the protection ratio fall below the required protection ratio threshold. A network consisting of M channels and N SUs is assumed. 


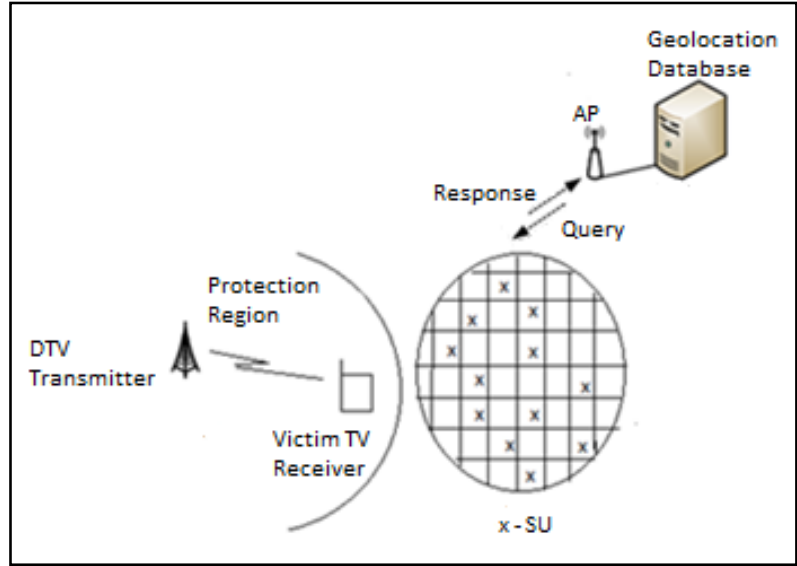

Figure 1: Interference scenario

\subsection{Problem Formulation}

The optimization problem is as follows [7]:

\section{Problem 1}

$p^{*}=\arg \min \emptyset(p)$

Subject to $C: p_{\min } \leq p_{i} \leq p_{\max }$.

$$
\emptyset(p)=\varphi(p)+c_{s} \sum_{i=1}^{N} \max \left[0, g_{i}^{s}\right]^{2}+c_{p} \max \left[0, g_{i}^{p}\right]^{2} .
$$

The optimization problem in Problem 1 is about minimization of sum power and minimization of interference threshold violations at SUs and at the PU. In equation (2), the first term, $\varphi(\mathrm{p})$, represents the sum power of all SUs, the second term $\left(c_{s} \sum_{i=1}^{\mathrm{N}} \max \left[0, g_{i}^{s}\right]^{2}\right)$ represents interference threshold violation for SUs while the third term represents interference threshold violation for PU. The terms $c_{s}$ and $c_{p}$ are penalty factors for SU interference threshold violation and PU interference threshold violation.

\subsection{Power Allocation Using Hybrid Firefly Algorithm, Particle Swarm Optimization and Genetic Algorithm}

This sub section presents the design of hybrid firefly algorithm, particle swarm optimization and genetic algorithm (FAGAPSO) for power allocation in a TVWS network. The hybrid algorithm takes into consideration concepts of PSO and GA proposed in [17] [15] and [16]. As described in [19], instead of FA starting with a random solution, it will have an initial solution as the solution found by PSO. As noted earlier, the final solution of FA depends on quality of initial solution. In addition to making use of initial solution of PSO, the proposed algorithm also makes use of PSO concepts of personal best and global best. Firefly movement will involve movement towards the global best solution and personal best solution. The algorithm also makes use of GA concept of crossover that helps to improve solutions of different chromosomes iteratively through mixing of solutions in different chromosomes.

The algorithm steps are outlined in Algorithm 1. In step 1 of Algorithm 1, optimal power allocation is first found using PSO. In step 2, FA starts with initial solution of PSO generated in Step 1. All fireflies will be initiated with solutions found in PSO particles at the end of PSO in Step 1. In step 3, after ranking fireflies according to their fitness, the best two fireflies are crossed over to generate four new offsprings. The four new offsprings are then ranked according to their fitness. The current best firefly will then be replaced by the best offspring if its fitness as measured by equation (1) is lower (better) than that of the best offspring. Firefly movement will involve a search towards local personal best and global best according to equation (2). The proposed algorithm therefore makes use some PSO operators including $P_{\text {best }}, P g_{\text {best }}, c_{1}$ and $c_{2}$.

$$
\begin{gathered}
x_{i}^{t+1}=x_{i}^{t}+c_{1} e^{-\gamma r_{i j}^{2}}\left(p_{i}-x_{i}^{t}\right)+c_{2} e^{-\gamma r_{i j}^{2}}\left(p_{g}-x_{i}^{t}\right) \\
+\alpha_{t} \epsilon_{t}^{i},
\end{gathered}
$$

\section{SIMULATION SET UP}

Parameters used in the simulation are outlined in Table I. Simulation was done using Matlab R2016a. Matlab is chosen because it is rich in in-built functions. SUs $(\mathrm{N}=1000)$ are distributed over an area of $1 \mathrm{~km}^{2}$. Fig. 2 shows the network diagram generated in Matlab. The channels to be considered are the ones in Nairobi central business district shown in Fig.3 Initially SUs are distributed across 10 channels i.e $\mathrm{M}=10$. Initial spectrum assignment is also done randomly. The free space path loss model was used to model path loss [24]:

$$
P L(d)=20 \log (d)+20 \log (f)-147.55,
$$

where $\mathrm{d}$ is the distance in meters and $\mathrm{f}$ is the frequency of operation. The proposed resource allocation algorithm is then used to assign power and spectrum to SUs.

Parameters used for FA are as follows: $\beta_{o}=1, \alpha=30$, $\gamma=10$, number of fireflies $\mathrm{NP}=20$. Parameters used for PSO are as follows: number of particles $=50$, inertia weights: $w_{\max }=4, w_{\min }=2$, social parameter $\mathrm{c}_{1}=2$ and cognitive parameter $c_{2}=2$. Parameters used for GA are as follows: number of chromosomes $=50$, mutation rate $=0.8$ and selection rate $=0.5$.

\section{PERFORMANCE EVALUATION}

In this section, simulation results for optimization of spectrum allocation using a variety of hybrid FA, PSO and GA algorithms are presented and discussed. FAGAPSO is compared with:

- FA, GA, PSO

- FAPSO1: FA with initial solution of PSO

- FAPSO2: FA with PSO operators i.e firefly movement using $P_{\text {best }}, g_{\text {best }}, c_{1}$ and $c_{2}$ as expressed in equation 3

- FAPSO3: FA with PSO operators with initial solution of PSO

- FAGA: Firefly algorithm with the use crossover feature of GA according to Step 3 of Algorithm 1

Simulation results are generated for 10 simulation runs and an average is done. The performance of the algorithms is compared using the following metrics: objective function value, sum throughput, PU SINR and SU SINR. 


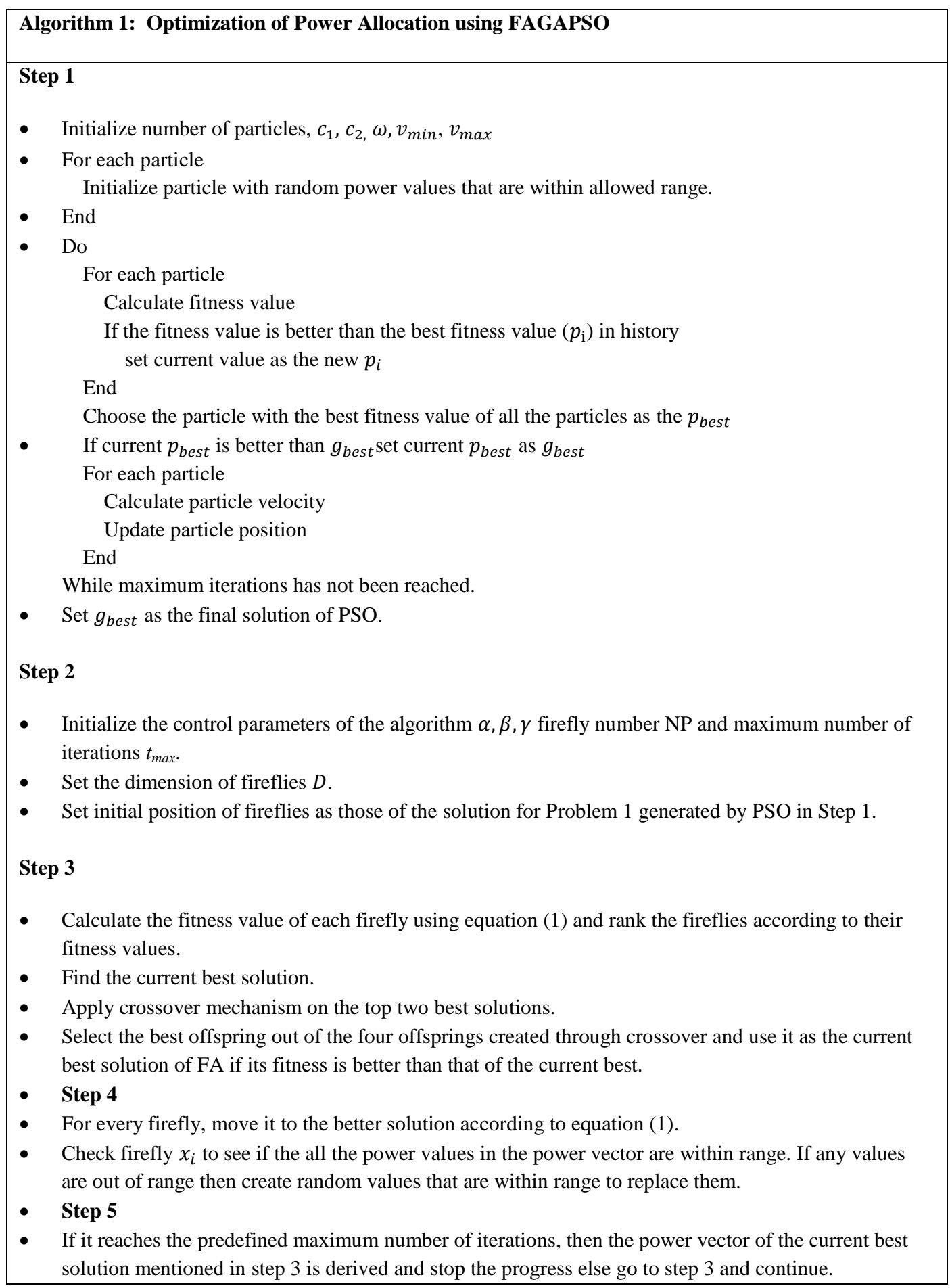

Table 1: Simulation Parameters

\begin{tabular}{|c|c|c|}
\hline Parameter & Value & Description \\
\hline$b_{m}$ & $8 \mathrm{MHz}$ & Bandwidth of TV channel \\
\hline $\mathrm{f}_{\mathrm{a}}$ & $694 \mathrm{MHz}$ & Centre frequency of DTV signal \\
\hline $\mathrm{P}_{\mathrm{TV}}$ & $-100 \mathrm{dBm}$ & $\begin{array}{c}\text { Power of DTV signal at victim } \\
\text { TV receiver }\end{array}$ \\
\hline$\delta_{\mathrm{n}}^{2}$ & $-102 \mathrm{dBm}$ & Noise power \\
\hline$\omega_{\mathrm{o}}$ & $23 \mathrm{~dB}$ & TV receiver SINR threshold \\
\hline
\end{tabular}

\begin{tabular}{|c|c|c|}
\hline Parameter & Value & Description \\
\hline$\rho_{\mathrm{o}}$ & $7 \mathrm{~dB}$ & SU SINR threshold \\
\hline $\mathrm{P}^{\mathrm{BS}}$ & $\begin{array}{l}36 \mathrm{dBm} \\
(4 \mathrm{~W})\end{array}$ & Transmit power of base station \\
\hline$p_{\max }$ & $30 \mathrm{dBm}$ & Maximum SU transmit power \\
\hline$\mu\left(c_{\mathrm{TV}}, \mathrm{c}_{\mathrm{n}}\right)$ & $0,-28 \mathrm{~dB}$ & $\begin{array}{c}\text { Adjacent channel interference } \\
\text { co-efficient }\end{array}$ \\
\hline $\mathrm{G}_{\mathrm{SU}}$ & $10 \mathrm{~dB}$ & SU antenna gain \\
\hline $\mathrm{G}_{\mathrm{PU}}$ & $10 \mathrm{~dB}$ & PU antenna gain \\
\hline
\end{tabular}




\begin{tabular}{|c|c|c|}
\hline Parameter & Value & Description \\
\hline $\mathrm{G}_{\mathrm{BS}}$ & $10 \mathrm{~dB}$ & Access point antenna gain \\
\hline$\beta_{\mathrm{o}}$ & 1 & FA parameter \\
\hline$\alpha$ & 30 & FA parameter \\
\hline$\gamma$ & 10 & FA parameter \\
\hline $\mathrm{NP}$ & 20 & Penalty term for SU interference \\
\hline $\mathrm{c}_{\mathrm{s}}$ & 1000 & Penalty term for PU interference \\
\hline $\mathrm{c}_{\mathrm{p}}$ & 1000 & \\
\hline
\end{tabular}

power allocation that minimizes interference in the network. According to Shannon channel capacity theorem, reduction in interference improves throughput. FAPSOGA improves sum throughput by $142 \%, 131 \%$ and $147 \%$ when compared to conventional GA, PSO and FA, respectively. The algorithm also achieves higher sum throughput compared to FAGA, FAPSO1, FAPSO2 and FAPSO3.

Table 2: Comparison of Sum Power in Network

\begin{tabular}{|c|c|c|}
\hline Algorithm & Sum Power (Watts) & $\begin{array}{c}\text { Percentage } \\
\text { Improvement }\end{array}$ \\
\hline FAPSOGA & 240 & \\
\hline GA & 1849 & $87 \%$ \\
\hline PSO & 1639 & $85 \%$ \\
\hline FA & 1842 & $87 \%$ \\
\hline FAPSO1 & 1318 & $82 \%$ \\
\hline FAPSO2 & 551 & $56 \%$ \\
\hline FAPSO3 & 253 & $5 \%$ \\
\hline FAGA & 1798 & $87 \%$ \\
\hline
\end{tabular}

Table 3: Comparison of Sum Throughput

\begin{tabular}{|c|c|c|}
\hline Algorithm & $\begin{array}{c}\text { Sum Throughput } \\
(\mathbf{G b} / \mathbf{s})\end{array}$ & $\begin{array}{c}\text { Percentage } \\
\text { Improvement }\end{array}$ \\
\hline FAPSOGA & 15.92 & \\
\hline GA & 6.57 & $142 \%$ \\
\hline PSO & 6.89 & $131 \%$ \\
\hline FA & 6.45 & $147 \%$ \\
\hline FAPSO1 & 7.73 & $106 \%$ \\
\hline FAPSO2 & 12.77 & $25 \%$ \\
\hline FAPSO3 & 15 & $6 \%$ \\
\hline FAGA & 6.49 & $145 \%$ \\
\hline
\end{tabular}

\subsection{Percentage of SUs less than SU SINR Threshold}

Table 4 shows comparison of the proposed algorithm with the rest of the algorithms in terms of percentage of SUs with SU SINR less than required threshold of $7 \mathrm{~dB}$ in the network. The results show that the FAGAPSO achieves the lowest percentage of SUs with SU SINR below threshold. This is because of the improved power allocation that minimizes interference in the network.

Table 4: Comparison of Percentage of SUs less than SU SINR Threshold

\begin{tabular}{|c|c|}
\hline Algorithm & $\begin{array}{l}\text { \% of SUs Less Than } \\
\text { SU SINR Threshold }\end{array}$ \\
\hline FAPSOGA & 19.9 \\
\hline GA & 36.36 \\
\hline PSO & 36.31 \\
\hline FA & 36.7 \\
\hline
\end{tabular}




\begin{tabular}{|c|c|}
\hline FAPSO1 & 35.01 \\
\hline FAPSO2 & 25.38 \\
\hline FAPSO3 & 19.85 \\
\hline FAGA & 36.25 \\
\hline
\end{tabular}

\subsection{Objective Function Value}

Table 5 shows comparison of the proposed algorithm with the rest of the algorithms in terms of achieved objective function value. The results show that FAGAPSO achieves the best (lowest) objective function value represented by equation (3). FAGAPSO improves objective function value by $77 \%, 68 \%$ and $77 \%$ when compared to conventional GA, PSO and FA, respectively. The algorithm is also better compared to FAGA and the other three FAPSO hybrid algorithms under consideration.

Table 5: Comparison of Objective Function Values

\begin{tabular}{|c|c|c|}
\hline Algorithm & $\begin{array}{c}\text { Objective Function } \\
\text { Value }\end{array}$ & $\begin{array}{c}\text { Percentage } \\
\text { Improvement }\end{array}$ \\
\hline FAPSOGA & 4691 & \\
\hline GA & 20783 & $77 \%$ \\
\hline PSO & 14842 & $68 \%$ \\
\hline FA & 20714 & $77 \%$ \\
\hline FAPSO1 & 15469 & $70 \%$ \\
\hline FAPSO2 & 7803 & $40 \%$ \\
\hline FAPSO3 & 4831 & $3 \%$ \\
\hline FAGA & 20273 & $77 \%$ \\
\hline
\end{tabular}

\subsection{Running time}

Table 6 shows comparison running time FAGAPSO with other algorithms. The run time in the table is for $200 \mathrm{SUs}$ in a network and 100 iterations for FA, GA and PSO. For FAGAPSO the number of iterations used for FA is set to 50 (half of iterations used by pure FA) while number of iterations used for PSO are 50 (half of iterations used for pure PSO). Simulation results show that the run time of FAGAPSO is higher than that of PSO and GA but lower than that of FA. It is also lower than that of other hybrid algorithms except FAPSO3.

\subsection{Analysis of Performance of the Algorithms}

FAGA has poor performance compared to the FAPSO1, FAPSO2 and FAPSO3. It can be seen from the results PSO is able to search the solution space for a continuous optimization problem better than both GA and FA. This makes a hybrid FA and PSO perform better than hybrid of FA and GA. Furthermore the use of crossover feature in FA only improves FA's exploration ability only but not exploitation ability.

Table 6: Comparison of Algorithm Running Time

\begin{tabular}{|c|c|}
\hline Algorithm & Time (Seconds) \\
\hline FAPSOGA & 8.73 \\
\hline GA & 4.38 \\
\hline
\end{tabular}

\begin{tabular}{|c|c|}
\hline PSO & 4.55 \\
\hline FA & 15.47 \\
\hline FAPSO1 & 10.16 \\
\hline FAPSO2 & 10.84 \\
\hline FAPSO3 & 7.66 \\
\hline FAGA & 11.47 \\
\hline
\end{tabular}

FAPSO2 performs better than FAPSO1. This implies that the use of PSO operators in FA allows hybrid FA get a better solution compared to using initial solution generated in PSO in FA. This is because the use of PSO operators improves FA's exploration and exploitation ability.

FAPSO3 performs better than FA, GA, PSO, FAPSO1 and FAPSO2 in all the performance metrics. This is because a hybrid FA with the use of initial solution of PSO as well the use PSO operators ( $P_{\text {best }}, g_{\text {best }}, c_{1}$ and $c_{2}$ as expressed in equation 2) enables FA to search the solution space better compared with FA, GA, PSO, FAPSO1 and FAPSO2 through improved exploration and exploitation ability.

Compared to other algorithms, FAGAPSO improves all the performance metrics of power allocation except percentage of SUs with SU SINR less than required threshold where it closely matches that of FAPSO3. The improvement in performance metrics is because the use of crossover feature of GA to mix top ranked fireflies in addition to the use of initial solution of PSO as well as PSO operators in FA further allows it to search the solution space better through further improved exploration ability. This enables FAGAPSO to generate highest sum throughput as well as the lowest sum power and objective function value.

\section{CONCLUSION}

The results have shown that FAGAPSO is better than FA as well as GA and PSO. Results have also shown that FAGAPSO is also better than FAGA as well as the three versions of FAPSO. Only FAPSO3 is closer to FAGAPSO. The only disadvantage of FAGAPSO is the slightly higher running time compared to GA and PSO. However, the slightly higher running time can be tolerated for improved power allocation that improves SU throughput and SU SINR. Future work includes testing the algorithm in a real world TVWS network.

\section{REFERENCES}

[1] K. Patil, R. Prasad, and K. Skouby, "A survey of worldwide spectrum occupancy measurement campaigns for cognitive radio," in Devices and Communications (ICDeCom), 2011 International Conference on, 2011, pp. $1-5$.

[2] M. Mehdawi, N. Riley, K. Paulson, A. Fanan, and M. Ammar, "Spectrum occupancy survey in HULL-UK for cognitive radio applications: measurement \& analysis," Int. J. Sci. Technol. Res., vol. 2, no. 4, pp. 231-236, 2013.

[3] M. Nekovee, T. Irnich, and J. Karlsson, "Worldwide trends in regulation of secondary access to white spaces using cognitive radio," Wirel. Commun. IEEE, vol. 19, no. 4, pp. 32-40, 2012.

[4] M. Fadda, V. Popescu, M. Murroni, P. Angueira, and J. Morgade, "On the Feasibility of Unlicensed 
Communications in the TV White Space: Field Measurements in the UHF Band," Int. J. Digit. Multimed. Broadcast., vol. 2015, pp. 1-8, 2015.

[5] J. Heo, G. Noh, S. Park, S. Lim, E. Kim, and D. Hong, "Mobile TV White Space with Multi-Region Based Mobility Procedure," IEEE Wirel. Commun. Lett., vol. 1, no. 6, pp. 569-572, Dec. 2012.

[6] M. Nekovee, "A Survey of Cognitive Radio Access to TV White Spaces," Int. J. Digit. Multimed. Broadcast., vol. 2010, pp. 1-11, 2010

[7] R. Kennedy, K. George, O.-O. William, O. Thomas, and O. Tonny, "Firefly algorithm based power control in wireless TV white space network," in AFRICON, 2017 IEEE, 2017, pp. 155-160.

[8] X.-S. Yang, "Firefly algorithms for multimodal optimization," in International Symposium on Stochastic Algorithms, 2009, pp. 169-178.

[9] S. Arora and S. Singh, "A conceptual comparison of firefly algorithm, bat algorithm and cuckoo search," in 2013 International Conference on Control, Computing, Communication and Materials (ICCCCM), Allahabad, India, 2013, pp. 1-4.

[10] K. Ronoh, G. Kamucha, T. Olwal, and T. Omwansa, "Improved Resource Allocation for TV White Space Network Based on Modified Firefly Algorithm," J. Comput. Inf. Technol., vol. 26, no. 3, pp. 167-167, Sep. 2018.

[11] S. Y. Lee, M. K. Kwon, and S. H. Lee, "Transmit power control scheme for TV white space wireless system," in Advanced Communication Technology (ICACT), 2011 13th International Conference on, 2011, pp. 1025-1029.

[12] Z. Xue, "Geolocation Spectrum Database Assisted Coexistence of Multiple Device-to-device in TV White Space," J. Inf. Comput. Sci., vol. 12, no. 11, pp. 44434456, Jul. 2015.

[13] H. R. Karimi, "Geolocation databases for white space devices in the UHF TV bands: Specification of maximum permitted emission levels," in New Frontiers in Dynamic Spectrum Access Networks (DySPAN), 2011 IEEE Symposium on, 2011, pp. 443-454.

[14] Z. Xue, L. Shen, G. Ding, Q. Wu, L. Zhang, and Q. Wang, "Coexistence among Device-to-Device communications in TV white space based on geolocation database," in High Mobility Wireless Communications (HMWC), 2014 International Workshop on, 2014, pp. $17-22$.

[15] S. Arunachalam, T. AgnesBhomila, and M. Ramesh Babu, "Hybrid Particle Swarm Optimization Algorithm and Firefly Algorithm Based Combined Economic and Emission Dispatch Including Valve Point Effect," in Swarm, Evolutionary, and Memetic Computing, vol 8947, B. K. Panigrahi, P. N. Suganthan, and S. Das, Eds. Cham: Springer International Publishing, 2015, pp. 647 660.

[16] P. Kora and K. S. Rama Krishna, "Hybrid firefly and Particle Swarm Optimization algorithm for the detection of Bundle Branch Block,” Int. J. Cardiovasc. Acad., vol. 2, no. 1, pp. 44-48, Mar. 2016.

[17] I. Fister, I. Fister, X.-S. Yang, and J. Brest, "A comprehensive review of firefly algorithms," Swarm Evol. Comput., vol. 13, pp. 34-46, Dec. 2013.

[18] J. Luthra and S. K. Pal, "A hybrid Firefly Algorithm using genetic operators for the cryptanalysis of a monoalphabetic substitution cipher," in 2011 World Congress on Information and Communication Technologies, Mumbai, India, 2011, pp. 202-206.

[19] R. Kennedy, O. Tonny, and K. George, "Novel Resource Allocation Algorithm for TV White Space Networks Using Hybrid Firefly Algorithm," Int. J. Comput., vol. 32 , no. 1, p. 20, 2019.

[20] National Institute of Technology, Warangal, India, B. V. Kumar, N. V. Srikanth, and Associate Professor, Department of Electrical Engineering, National In stitute of Technology, Warangal, India, "Bat Algorithm a nd Firefly Algorithm f or Improving Dynamic Stability of Power Systems Using UPFC," Int. J. Electr. Eng. Inform., vol. 8, no. 1, pp. 164-188, Mar. 2016.

[21] M. Elkhechafi, H. Hachimi, and Y. Elkettani, "A new hybrid cuckoo search and firefly optimization," Monte Carlo Methods Appl., vol. 24, no. 1, pp. 71-77, Mar. 2018.

[22] A. Layeb and Z. Benayad, "A Novel Firefly Algorithm Based Ant Colony Optimization For Solving Combinatorial Optimization ProblemS,” Int. J. Comput. Sci. Appl., vol. 11, no. 2, p. 19, 2014

[23] D. Gurney, G. Buchwald, L. Ecklund, S. Kuffner, and J. Grosspietsch, "Geo-location database techniques for incumbent protection in the TV white space," in New Frontiers in Dynamic Spectrum Access Networks, 2008. DySPAN 2008. 3rd IEEE Symposium on, 2008, pp. 1-9.

[24] O. Katircioglu, H. Isel, O. Ceylan, F. Taraktas, and H. B. Yagci, "Comparing ray tracing, free space path loss and logarithmic distance path loss models in success of indoor localization with RSSI," in 2011 19thTelecommunications Forum (TELFOR) Proceedings of Papers, Belgrade, Serbia, 2011, pp. 313-316. 